\title{
Mechanische Fest/Flüssig-Trennung im Wandel der Zeit
}

HARALD ANLAUF*

\section{Querschnittstechnologie von A bis Z}

Kaum ein Gebiet der mechanischen Verfahrenstechnik durchdringt in einer solchen Vielfalt und Selbstverständlichkeit die unterschiedlichsten Bereiche unserer Lebens- und Produktionsumgebung, wie die Abtrennung von festen Partikeln aus Flüssigkeiten. Bei der Reinigung von Abwässern und der Gewinnung von Trinkwasser zum Beispiel erfüllt die Fest/Flüssig-Trennung Grundbedürfnisse unserer Existenz.

Oft als unspektakulär am Ende eines Produktionsprozesses angeordnete Notwendigkeit, wird die Bedeutung der Trenntechnik mitunter auch verkannt. Wer denkt beispielsweise bei biotechnologisch mit hochspezialisierten Mikroorganismen erzeugten Wirkstoffen daran, dass ca. 50 - $90 \%$ der Herstellkosten durch die Aufbereitungsschritte im sogenannten Down-stream Processing und damit im Wesentlichen durch Trenntechnik bestimmt werden?

Als typische Querschnittstechnologie ist die mechanische Fest/Flüssig-Trennung von A, wie Abwasserreinigung über $\mathrm{B}$, wie Bioproduktgewinnung quer durch das ganze Alphabet bis Z, wie Zuckerraffinierung zu finden.

Partikeldurchmesser von mehreren Zentimetern bis hinab zu wenigen Nanometern, Konzentrationen von wenigen Teilchen in der Flüssigkeit bis hin zum nicht mehr fließfähigen Schlamm, abrasive Mineralteilchen und empfindliche Zellkulturen, in weiten Bereichen variierende $\mathrm{pH}$ Werte, Viskositäten und andere physiko-chemische Eigenschaften der Suspensionsflüssigkeiten, Mengenströme von wenigen Millilitern bis hin zu tausenden von Kubikmetern pro Stunde sowie unterschiedlichste Anforderungen an das Trennergebnis haben im Laufe der Geschichte zu einer fast nicht mehr zu überblickenden Vielfalt an Trennverfahren und Trenngeräten geführt.

\section{Historische Entwicklung}

Schon früh bedienten sich die Menschen den beiden durch die Natur vorgegebenen trenntechnischen Grundprinzipien der Sedimentation und der Filtration, die sich unter dem Einfluss der Schwerkraft bei jedem Absetzvorgang von in Wasser aufgewirbelten Teilchen oder bei der Klärung von Wasser mittels Drainage durch poröse Sand- oder Kießschichten beobachten lassen.

Den als "Sihr" bezeichneten und auch heute noch bekannten porösen Tongefäßen zur Filtration von Trink- wasser, Wein und anderer Flüssigkeiten bedienten sich schon die alten Ägypter. Mehrere tausend Jahre alte bronzene Kolatorien wurden bei Theben gefunden, in die als Filtermittel Binsen- oder Ginsterschichten eingelegt waren. Diese Technik kultivierten die Römer zur Filtration von Wein u. a. Etwa 100 n. Chr. beschreibt der in der Han-Dynastie lebende Chinese TSAI-LUN ein Verfahren zur Herstellung von Filterpapier. Die mittelalterlichen Alchimisten bedienten sich bereits der Holzkohle als Filter- und Entfärbungsmittel. Aus dem 18. und 19. Jahrhundert sind die ersten Experimente mit natürlichen Membranen, wie Schweins- und Fischblasen, dokumentiert. 1748 wurden Experimente des französichen ABBÉ J. A. NOLLET zur Osmose veröffentlicht. Ein stürmischer Aufschwung auch in der Trenntechnik setzte mit der Industrialisierung in der Mitte des 19. Jahrhunderts ein. Die jetzt schnell wachsende Zahl zu lösender Trennprobleme und höhere Anforderungen an die Trennergebnisse erforderten neue Technologien. Der allgemeine technische Fortschritt erschloss den Trennverfahren über die Nutzung von Schwerkraft und mechanischen Presskräften hinaus die Anwendung von Vakuum, Druckluft, hydraulischem Flüssigkeitsdruck und Zentrifugalkräften. Zwischen 1850 und 1860 wurde in England die Filterpresse in der auch heute noch prinzipiell gültigen Konstruktionsweise entwickelt. Bereits 1932 existierten auf diesem Gebiet 1500 Patente. 1891 kam Kieselgur als Hilfsmittel zur Klärfiltration zum Einsatz. 1907 wurde von ERNST JAHN mit der „Trennschleuder" die erste konische GegenstromDekantierzentrifuge patentiert, mit der zunächst die industrielle Gewinnung von Stärke entscheidend verbessert und beschleunigt werden konnte (s. Abb. 1).

Abbildung 1.

Dekanter mit vollkonischer Trommel (Bauart Jahn).

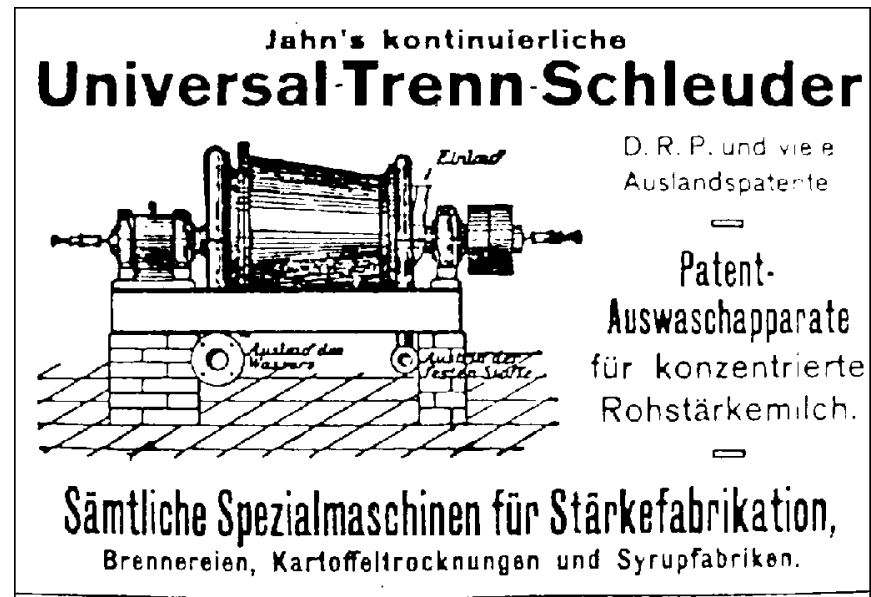

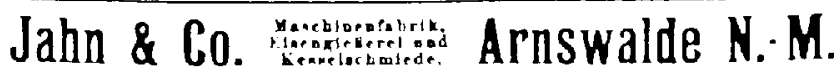

* Dr.-Ing. H. ANLAUF (E-mail:

harald.anlauf@mvm.uni-karlsruhe.de)

Universität Karlsruhe ( $\mathrm{TH}$ ), Institut für

Mechanische Verfahrenstechnik und

Mechanik, D-76128 Karlsruhe. 
1915 entstehen die ersten Tiefenfilterschichten aus faserförmigen Stoffen zur Entkeimungsfiltration. Zwischen 1914 und 1918 legten ZSIGMONDY und BACHMANN in Göttingen den Grundstein für die industrielle Produktion von definierten mikroporösen Membranen aus Cellulosedrivaten. Thren industriellen Durchbruch und die Entwicklung zu einer der Schlüsseltechnologien für die Abtrennung feinster Teilchen vollzog die Membranfiltrationstechnik aber erst in den 60er und 70er Jahren des vorigen Jahrhunderts mit neuen Verfahren zur Herstellung aus diversen Polymerwerkstoffen, Keramika u. a.

\section{Entwicklungsmechanismen}

In Analogie zur belebten Natur könnte man, wie es Prof. TRAWINSKI aus Hirschau vor etwa 10 Jahren einmal formulierte, die Trennverfahren als einem ständigen evolutionären Entwicklungsprozess unterworfen sehen, der durch Mutation und Selektion vorangetrieben wird. Ausgelöst wird die Entwicklung durch den Druck von Erfordernissen in einem sich verändernden Umfeld, wie etwa der Energiekriese vor 30 Jahren, neuen Umweltschutzbestimmungen oder neuen Anforderungen an die Qualität von Produkten. Die durch eine „zündende Idee“ ausgelöste apparative Mutation führt zu einem modifizierten oder ganz neuen Apparat. Dieser muss sich dann am Markt als besser oder schlechter an die Verhältnisse angepasste Spezies erweisen, setzt sich durch oder verschwindet wieder. Um sich erfolgreich entwickeln und bestehen zu können, benötigt ein Trennverfahren allerdings neben dem Bedarf auch das geeignete werkstofftechnische Umfeld. Viele der schon im ersten Drittel des vorigen Jahrhunderts geborenen Ideen verschwanden daher wieder im Archiv und konnten erst in jüngerer oder jüngster Zeit realisiert und in die Praxis eingeführt werden.

\section{Entwicklung durch Sprung auf ein neues Prinzip}

So boten beispielsweise erst die erwähnte Energiekrise und die zu dieser Zeit verfügbaren modernen Werkstoffe das Umfeld, in dem sich die mit dem Namen STAHL verbundene „zündende Idee“ der Hyperbarfiltrationstechnik erfolgreich in Karlsruhe entwickeln und weltweit einführen konnte (s. Abb. 2).

Die Begrenzung der Druckdifferenz herkömmlicher Vakuumtrommel- und -scheibenfilter konnte durch die Einhausung dieser konstruktiv ausgereiften Filtertypen erfolgreich aufgehoben und der Überdruckfiltration bis ca. $8 \mathrm{MPa}$ zugänglich gemacht werden. Insbesondere in der Rohstoffaufbereitung mit großen Mengenströmen konnten damit hohe thermische Trocknungskosten drastisch reduziert oder ganz vermieden werden. In jüngster Zeit ist durch die zusätzliche Anwendung von überhitztem Wasserdampf im Druckbehälter die inzwischen klassische Hyperbarfiltration zur Dampfdruckfiltration weiterentwickelt worden. Dieses mechanisch/thermische Verfahren nutzt Synergien aus der Kombination von mechanischer Entfeuchtung und thermischer Trocknung des Filterkuchens.
Abbildung 2.

Hyperbar-Scheibenfilter (Bauart Andritz).

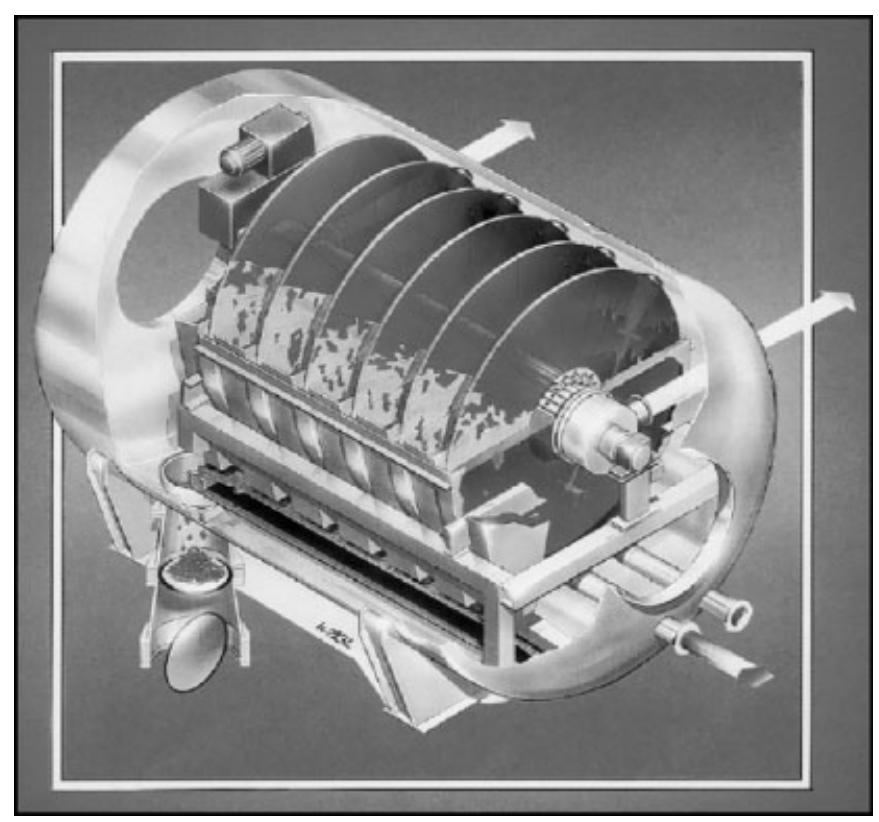

\section{Entwicklung durch kontinuierliche Verbesserung eines Grundprinzipes}

Als Beispiel soll hier die schon erwähnte Filterpresse als typische Vertreterin der weitverzweigten Familie der Druckund Pressfilterapparate dienen. In den ersten Ausführungen als Rahmenfilterpresse besaßen diese Apparate gusseiserne Gestelle, einen Spindelverschluss und hölzerne Rahmen und Platten, die mit Baumwolltüchern als Filtermedium bespannt waren. Bereits 1918 erwähnte die Fa. DEHNE aus Halle an der Saale in ihrem Filterpressenkatalog 22000 ausgelieferte Apparate. Durch die Entwicklung neuer Trenngeräte, wie Vakuum- und Druckdrehfilter, Anschwemmfilter, Dekantierzentrifugen u. a. wurden die Filterpressen im Laufe der Zeit in einigen Industriebereichen zurückgedrängt. Dennoch und insbesondere durch ihre kontinuierliche Verbesserung und Automatisierung können auch heute noch viele Trennprobleme am besten mit einer Filterpresse gelöst werden. Meilensteine des Fortschrittes bei den Filterpressen lagen insbesondere in der Verbesserung des Kuchenaustrages und der immer weitergehenden Automatisierung (s. Abb. 3).

Abbildung 3.

Entwicklung der Filterpresse.

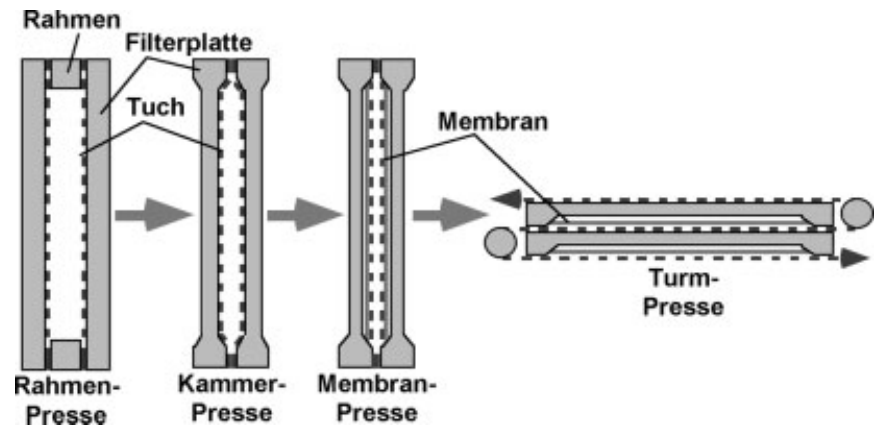


So brachte der Übergang von der Rahmenpresse, bei welcher der Filterkuchen mühsam aus Rahmen entfernt werden musste zur Kammerfilterpresse eine erhebliche Erleichterung des Kuchenaustrages. Die Einführung des hydraulischen Pressenverschlusses, die Möglichkeit zur Herstellung der Filterplatten aus Polymerwerkstoffen, der Einsatz oberflächenbehandelter Kunststofffiltergewebe und die Verbesserung der Filtrationseigenschaften schwer filtrierbarer Suspensionen durch polymere Flockungshilfsmittel ermöglichten den Bau großer Filtereinheiten bis ca. $1000 \mathrm{~m}^{2}$ Filterfläche bei bis zu 150 Platten und Plattenformaten bis $\mathrm{zu} 4 \mathrm{~m}^{2}$. Immer neue Vorrichtungen zur mechanisierten Unterstützung des Kuchenaustrages kamen hinzu. Einen weiteren Entwicklungssprung erfuhr die Filterpresse durch die Einführung der Membranfilterplatten, welche das mechanische Auspressen des Filterkuchens mit einer Pressmembran ermöglichten. Dies erlaubte geringe Speisedrücke, vollständige Restvolumenfiltration und besonders gleichmäßiges Auspressen bzw. Verdichten des Kuchens, was nicht nur für niedrige Restfeuchten sondern auch für ein Auswaschen des Feststoffes sehr vorteilhaft ist. Die Turmfilter-pressen der neuesten Generation mit horizontal angeordneten Membranfilterplatten und Kuchenauswurf durch Verfahren des Filtertuches verbindet schließlich die Vorteile der horizontalen Filtration auf einem Bandfilter mit dem hohen Filtrationsdruck der Filterpresse. Neben diesen kontinuierlichen Verbesserungen haben sich auf der Basis des Grundprinzipes auch einige „Derivate“ der Filterpresse, wie ein Schichtenfilter zur Tiefenfiltration, ein Rückspülfilter, die Filterpresse mit überlagerter Elektrofiltration oder die "heiße Filterpresse“ als mechanisch/thermischer Kombinationsapparat entwickelt.

\section{Entwicklung durch Anpassung eines Grundprinzipes an neue Aufgaben}

Die Dekantier- oder Vollmantelschneckenzentrifuge als Repräsentantin der Dichtetrennverfahren ist eines der in seinen verschiedenen Modifikationen am universellsten eingesetzten Fest/Flüssig-Trenngeräte (s. Abb.4).

Abbildung 4.

Gegenstrom-Dekantierzentrifuge (Bauart $\alpha$ ALFA LAVAL).

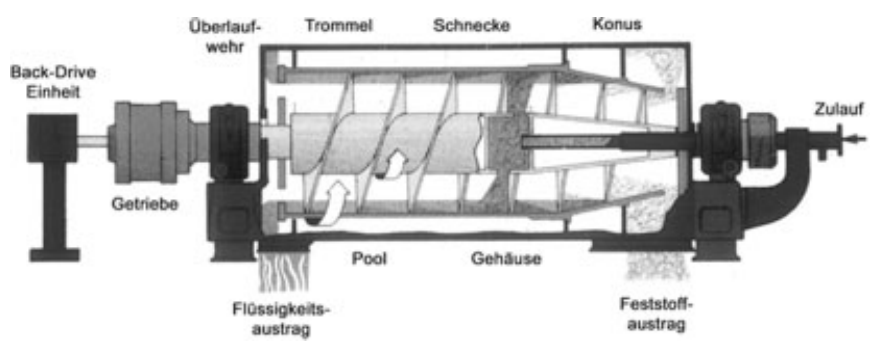

In der ursprünglichen Gegenstrombauweise mit zylindrisch-konischer Vollmanteltrommel stellt der Dekanter mit bis ca. 5000facher Erdbeschleunigung, Trommeldurchmessern bis zu 1,8 m und Suspensionsdurchsätzen bis hin zu ca. $250 \mathrm{~m}^{3} / \mathrm{h}$ ein außerordentlich vielseitig einsetzbares Trenngerät dar. Durch konstruktive Modifizierung konnte der Einsatzbereich dieses Maschinenprinzipes stark erweitert werden. So gibt es spezielle Dekanter für grobkörnige Produkte, wie etwa PVC und Bauarten für extrem feinkörnige Suspensionen, wie Abwasserschlämme. Es werden Eindickdekanter zur Aufkonzentrierung von Suspensionen und Dekanter für das weitgehende Auspressen kompressibler Schlämme gebaut. Mit Dekantern kann man Partikelverteilungen klassieren oder unterschiedliche Stoffe sortieren. Es ist möglich, mit Dekantern Dreiphasensysteme zu trennen oder Fest/Flüssig- bzw. Flüssig/Flüssig-Extraktionen zu realisieren. Vertikale Dekanter für hohe Temperaturen und hohe Drücke sowie Dekanter mit Siebteil zur Nachentfeuchtung körniger Stoffe durch Filtration werden gebaut.

\section{Trends und Zukunft}

Durch verschärfte Vorschriften werden immer kleinere Feststoffmengen und kleinere Teilchen aus großen Flüssigkeitsströmen abgetrennt werden müssen. Allein der europäische Markt im Bereich Wasser und Abwasser wird nach einer Prognose von Frost und Sullivan bis 2007 auf eine Summe von ca. 4 Mrd. Euro wachsen. Über die Hälfte der Umsätze in der Prozesswasseraufbereitung soll durch die stark wachsende Membrantechnik erzielt werden. Weitere neue Herausforderungen an die Trenntechnik ergeben sich durch die vermehrte Produktion nanoskaliger Feststoffe für die unterschiedlichsten Anwendungen. Die Erzeugung sehr spezifischer Produkteigenschaften in Hinblick auf Stoffreinheit, Unversehrtheit der Partikeln, Selektivität der Abscheidung u. a. erfordert spezifisch angepasste apparative Lösungen. Die Weiterentwicklung von mechanisch/ thermischen, mechanisch/elektrischen und mechanisch/ magnetischen Kombinationsverfahren wird hierzu Beiträge liefern. Die Verwendung neuer Materialien aus dem Bereich der Keramika und der verstärkten Kunststoffe sowie die Entwicklung von selbstreinigenden Oberflächen wird die Apparatetechnik verbessern. Durch weitere Fortschritte in der Inline-Messtechnik wird die Effizienz von Trennapparaten durch verbesserte Prozesssteuerung erhöht werden. Verwirklicht werden können diese Entwicklungen auf längere Sicht allerdings nur, wenn es durch weitere gezielte Förderung gelingt, die Erhaltung und Fortentwicklung der Expertise auf diesem traditionellen Gebiet der Verfahrenstechnik durch Ingenieursausbildung und Forschung zu gewährleisten.

\section{Literatur}

[1] R. Bott, T. Langeloh, Solid/Liquid Separation Lexicon, Wiley-VCH-Verlag, Weinheim 2002.

[2] H. Gasper, D. Oechsle, E. Pongratz, Handbuch der industriellen Fest/Flüssig-Filtration, Wiley-VCHVerlag, Weinheim 2000.

[3] S. Ripperger, Mikrofiltration mit Membranen, VCH-Verlag, Weinheim 1992.

[4] A. Rushton, A. S Ward, R. G. Holdich, Solid-Liquid Filtration and Separation Technology, VCH-Verlag, Weinheim 1996. 
Der Autor

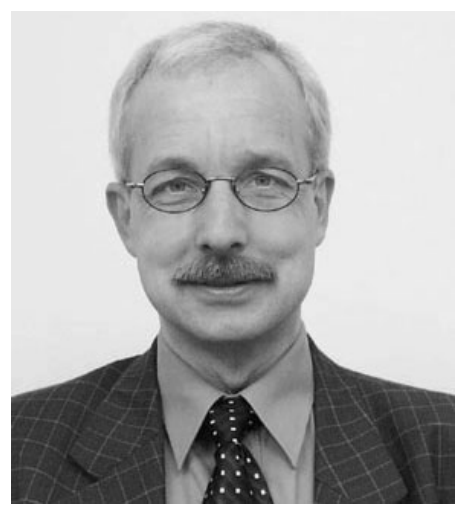

Harald Anlauf

HARALD ANLAUF wurde am 3.7.1954 in Berlin geboren. Nach einer klassisch humanistischen Schulausbildung mit Latein und Altgriechisch in Berlin verließ ich 1973 nach dem Abitur schweren Herzens aber neugierig auf die Welt das Elternhaus. Nach einem Betriebspraktikum bei der Siemens AG begann ich 1974 Chemieingenieurwesen in Karlsruhe zu studieren. Mein Chemielehrer hatte es in außergewöhnlicher Weise verstanden, Begeisterung für sein Fach zu wecken. Die Verbindung der Chemie mit dem Ingenieurwesen schien mir etwas Handfestes zu sein. Als ambitionierter Bastler und Sohn eines Bildhauers versprach ich mir auch viel vom kreativen Element des InGENIEurberufes. Beide Erwartungen haben sich für mich erfüllt.

Die Wahl des Hauptfaches Mechanische Verfahrenstechnik führte mich an das Institut von Prof. HANS RUMPF, wo ich meine Diplomarbeit beim gerade neu auf den Lehrstuhl berufenen Prof. WERner STAHL mit einem Thema zur Schälzentrifuge anfertigte. Als erster Doktorand hatte ich dann das Privileg, unter STAHL der Karlsruher Schule der Fest/Flüssig-Trennung vom Beginn angehören zu dürfen. In der Arbeitsgruppe Druckfiltration führten meine Grundlagenarbeiten 1985 zur Promotion. Fast zeitgleich ging die erste großindustrielle Hyperbarfiltrationsanlage in der deutschen Kohleindustrie in Betrieb. Ein Traum jedes Ingenieurs, den Weg einer innovativen Technologie mit allen Windungen und Wendungen von der zündenden Idee bis zur erfolgreichen Einführung in die industrielle Praxis begleitet zu haben, wurde für mich wahr. Die Vielseitigkeit der Tätigkeit, der Spaß an der Lehre und das besondere Arbeitsumfeld ließen mich das Angebot, an der Hochschule zu bleiben, annehmen und dem Institut für MVM bis heute die Treue halten. Durch meine Dienststellung kümmere ich mich um vielfältige administrative und organisatorische Aufgaben mit dem Ziel, ein gutes und effizientes Arbeitsum- feld für Forschung und Lehre bereitzustellen. Ein weiterer Schwerpunkt liegt in der aktiven Mitarbeit in Forschung und Lehre des Institutes bzw. der Fakultät für Chemieingenieurwesen und Verfahrenstechnik in Karlsruhe.

Ein darüber hinaus seit vielen Jahren an der École Supérieure de Biotechnologie in Straßburg bestehender Lehrauftrag, der Aufbau einer langjährigen Kooperation mit der Universidad Central de Venezuela in Caracas und viele internationale Projekte in China, Indien, Russland, Chile u. a. sind eine wertvolle Bereicherung insbesondere in Hinblick auf das Verstehen von Kultur und Lebensumständen der Menschen in anderen Teilen unserer Welt. Seit dem Jahr 2000 leite ich den VDI/GVC-Fachausschuss "Mechanische Flüssigkeitsabtrennung“ als wichtiges Bindeglied zwischen Hochschulforschung und industrieller Praxis. Durch die Organisation und die Mitwirkung an Fachveranstaltungen, Kongressen und Weiterbildungskursen für schon im Beruf stehende Ingenieure möchte ich einen Beitrag für eine lebendige Gestaltung des Austausches zwischen Theorie und Praxis leisten. Besonders wichtig ist mir hierbei die Förderung der Facharbeit auf europäischer und internationaler Ebene.

Neben dem Beruf gibt es natürlich auch ein Privatleben. Aus der während meiner Promotionszeit gegründeten Familie sind zwei Töchter hervorgegangen, die jetzt gerade ihr Studium aufnehmen. Ich bin begeisterter Marathonläufer, liebe Fahrradtouren und schätze den Karatesport. Ich koche sehr gern und kann bei einem guten Buch die Welt um mich herum vergessen. Für die berufliche $\mathrm{Zu}$ kunft wünsche ich mir wieder bessere materielle Randbedingungen für die vielen spannenden und wichtigen Forschungsaufgaben, die noch vor uns liegen und ich wünsche mir noch mehr junge Menschen, die sich von der Verfahrenstechnik begeistern lassen. 